\title{
Acquisition of Positional Accuracy with Comparative Analysis of GPS and EGNOS in Urban Constituency
}

\author{
Zeeshan $\mathrm{Ali}^{1}{ }^{*}$, Riaz Ahmed Soomro ${ }^{2}$, Faisal Ahmed Dahri ${ }^{3}$, Muhammad Mujtaba Shaikh ${ }^{4}$ \\ IREA-CNR (National Research Council of Italy), University of Naples "Parthenope", Napoli, Italy" \\ Department of Telecommunication Engineering, Mehran UET, Jamshoro, Pakistan ${ }^{2,3}$ \\ Department of Telecommunication Engineering, University of Malaga (UMA), Malaga, Spain $^{4}$
}

\begin{abstract}
Over the years, precise positioning has been the ultimate goal for Satellite Navigation Systems. The American Global Navigation Satellite System deliver the position and time information intended for various sectors such as vehicle tracking, oil exploration, atmospheric studies, astronomical telescope pointing, airport and harbor security tracking etc. Corresponding technological competitors such as Russian Global Navigation Satellite System (GLONASS), European Union's GALILEO, China's BeiDou and Japanese Quasi Zenith Satellite System (QZSS) are few other versions of Satellite based Augmentation Systems. Nevertheless, stern security measures, geographical statistics and stimulation of diverse Electronic Gadgets at indoor/outdoor surroundings make it critical to acquire data about any vicinity with seamless accessibility, accuracy and integrity with satellite links. In this paper, positional accuracy has been tested with analysis of EGNOS, EDAS and simple GPS receiver models at Rome City, Italy. To support results, various real time experiments/tests has been performed with GPS Receiver SIRF Demo software. The test was conducted on-board a car by installing a laptop equipped with GPS Receiver plus supportive SBAS (EGNOS particularly) through three diverse bus routes of locality and outcomes of few tested samples inside the Rome City center are specified to check the availability of desired satellite signals. Subsequently, comparative analysis has been executed between the simple GPS data received and GPS + EGNOS data collected during daytime traffic. The strength of test signals reveals accuracy of EGNOS in open terrain area with less congestion. Furthermore, Asian and European Advanced GPS systems are compared in terms of performance as well as feasibility of authentic, accurate and swift satellite navigation systems.
\end{abstract}

Keywords-Differential GPS; augmentation; EGNOS; EDAS; on-board equipment; urban and positional accuracy

\section{INTRODUCTION}

In Satellite Communication, Road Vehicle Navigation Systems has emerged potential technology in the domain of Intelligent Transport Systems. Subsequently numerous road applications such as traveler information, automatic emergency calls, route guidance, freight management, advanced driver assistance or electronic fee collection involve On-Board Equipment (OBE) capable of offering highly accurate location obtainable at low cost. To acquire the positional accuracy and integrity with Global Positioning System, EGNOS (European Geostationary Navigation Overlay Service) has been assimilated in European territory; distinctive package as it generates warning messages in case of positional error during satellite navigational calculations. Subsequently, it discards the satellites when false readings appear on display by evaluating certain threshold levels as outcomes [1]. SBAS is designed to grasp satellites navigation signals and broadcast GPS category of signals controlling integrity and wide-area differential correction augmentation data [2]. The EGNOS system superimposes over the GPS and GLONASS schemes to enrich accuracy, availability, reliability and continuity of positional estimation. SBAS concept is better approach in timely correct information of system for integration and correction to the random measurements which leads to the accuracy of coordinates [3]. GPS in urban and mountain areas, where GNSS signals are either blocked or degraded by natural or artificial obstacles, unable to provide accurate positioning due to the poor signal quality [4]. The AUS/NZ SBAS moreover broadcast exact satellite orbits and clock remedies to help ongoing drift equivocalness Precise Point Positioning (PPP) service that can convey $5-20 \mathrm{~cm}$ precision [5-8]. The authors have investigated DGPS and EGNOS receivers used vessel maundering in the bay of Gdansk. Two receivers were exploited to record the coordinates [9]. Two measurement sessions were adopted at fixed point to analyze the system performance. In determination of accuracy and integrity of positioning, different approaches were used to compute accurate and precise location. It is noted that variant in calculation of design which meets the integrity requirement of navigation system [10]. In this paper, the availability of effective, seamless and accurate signals has been analyzed by executing few experiments around a local bus route inside the Rome city centre to check the existence of signals during day time traffic. The combination of GPS+EGNOS could be suitable approach to cope up with an accurate positioning problem can improve the accuracy, continuity and integrity of positioning. The outcomes of practical tests confirmed the coverage of GPS and EGNOS signal in urban areas as well as detected the transmission time to a control centre (positioning data acquired from running vehicle). Moreover, the vehicle adopted several bus routes of public transport around the city of Rome.

The rest of the paper is structured as follows: the fundamentals of EGNOS framework is discussed in Section 2. The EDAS architecture and its performance services is explained in Section 3. The experimental setup of the proposed approach is described in Section 4. Section 5 reports the results and finally Section 6 concludes the paper.

*Corresponding Author 


\section{FUNDAMENTAL FRAMEWORK OF EGNOS}

The broadcasting of integrity messages and differential corrections of satellites has been accomplished with current GNSS setup; integrates by means of network of reference stations positioned across the globe having different names of Satellite Based Augmentation System (SBAS) in diverse constitutions (SBAS intensifies and stabilize the GNSS deployment) [11].

SBAS has been employed by various regions such as Western Europe and North Africa establish EGNOS whereas Wide Area Augmentation System (WAAS) by USA and Multi-Functional Satellite Augmentation System (MSAS) by Japan are corresponding satellite systems [12].

\section{A. EGNOS Structural Architecture}

EGNOS, Ranging and Integrity Monitoring Stations (RIMS) accumulate raw GPS data measurements and scrutinize signals quality, multipath mitigation and perceive satellite failure affairs in the processing centers. The user collects corrections and integrity statistics by means of PRN120 and PRN136 as geostationary satellites [13].

The functional skeleton of SBAS sub systems has been indicated in the Fig. 1 that summarizes GPS transmitted data (in some cases GLONASS satellites) to receiving customer. This navigated data has been monitored by networks controlled under SBAS service providers [13].

\section{B. Operation of the EGNOS Segments}

EGNOS signal is being broadcasted by the space segment that includes three GEO Space Vehicles (SVs) associated with a unique pseudo random noise; ground control segment manages the system as well as processes EGNOS signals simultaneously. After this activity, diverse categories of users are benefited with the development of EGNOS compliant receivers as the part of user segment. Fig. 2 shows the ground segment of EGNOS. In Europe, 41 Ranging and Integrity Monitoring (RIM) Stations are installed whereas few terminals are deployed at USA and North Africa [14].

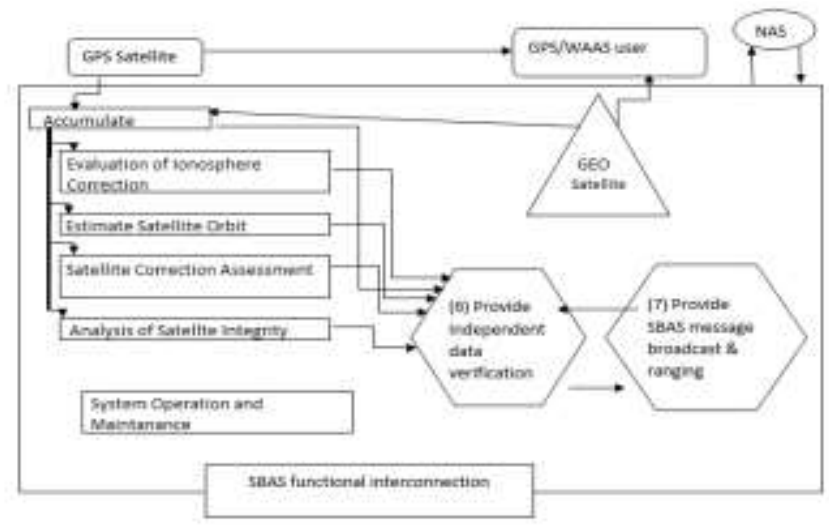

Fig. 1. SBAS Functional Overview.

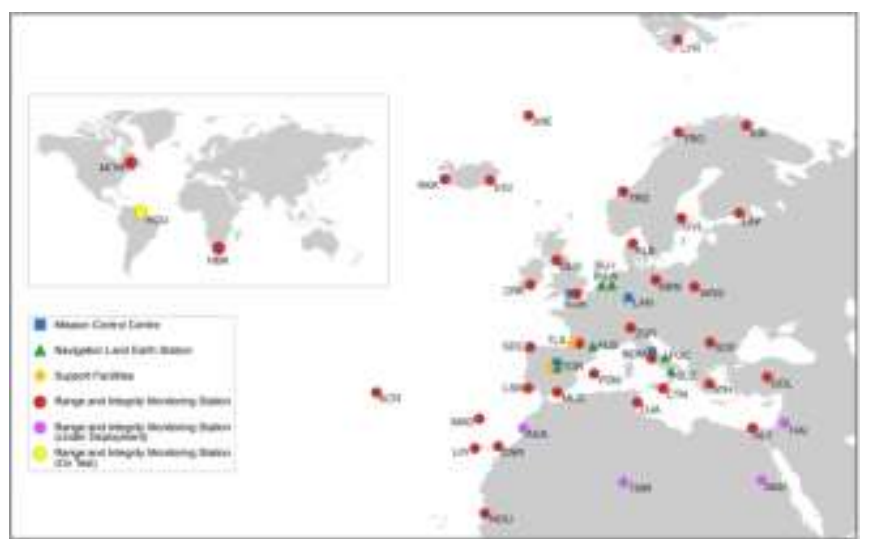

Fig. 2. EGNOS Ground Segment.

\section{EGNOS DATA ACCESS SERVICE (EDAS) SYSTEM ARCHITECTURE AND PERFORMANCE BASED SERVICES}

EGNOS delivers a terrestrial data service termed as EGNOS Data Access Service (EDAS) with real time data access (EGNOS) is achievable via ground transmission systems. File Transfer Protocol (FTP) offers EGNOS data to authorized customers (e.g., added-value application providers). Subsequently, EGNOS infrastructure (Navigation Land Earth Station and RIMS) acquires data with EDAS single point of access and utilize the real time data to specify performance boundaries [14]. Serially, satellite navigation data engendered by ground stations; EDAS permits users to "plug in" to EGNOS as indicated in Fig. 3 and Fig. 4 represents the EDAS service layout. The acquired data can be utilized efficiently in harsh environment, where signals distracted due to interference or when signals are blocked or invisible [14].

Correspondingly, EGNOS feeds the data to EGNOS Data Server (EDS) and execute number of tasks such as:

- Huge amount of customers is permitted by accepting feasible connection.

- Extra Security Layer has been initiated between customers and EGNOS.

- EGNOS registered data formats and protocols are secured plus EGNOS data has been processed via EDAS services.

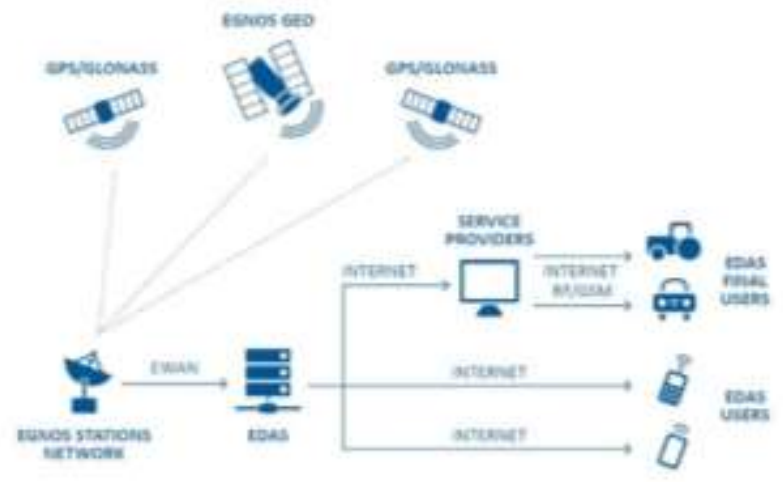

Fig. 3. EDAS High Level Architecture [14]. 


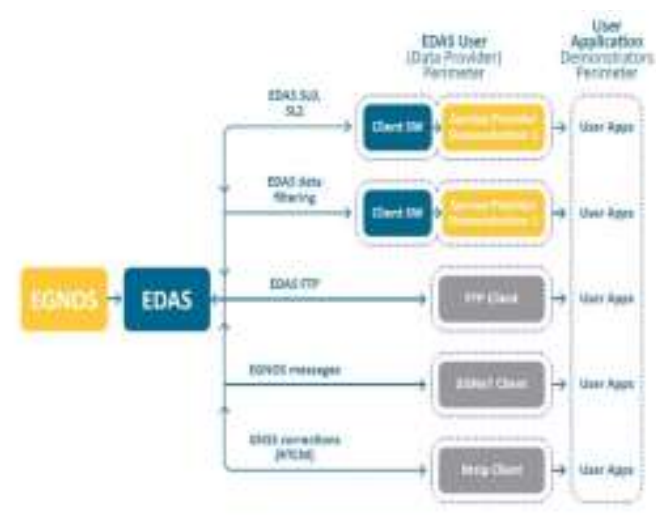

Fig. 4. EDAS Service Flow Diagram [14].

\section{EXPERIMENTS}

\section{A. Test Availability}

It has been evident in several FP7/ESA projects that EGNOS is capable enough to uplift the accuracy of GPS receiver in highways (former utilization of EGNOS/GPS receiver was based on transportation of hazardous items). Consequently, EGNOS messages are sent to the receiver via Geostationary Satellite. But, the availability of EGNOS signals via satellite seems to be lacking in the urban areas due to geographical and population dynamics. Therefore, Internet Connection through the EDAS service is an alternate way to deliver EGNOS messages in urban territory.

\section{B. Test Setup}

This test is conducted on-board with a GPS receiver connected with the laptop to support SBAS (EGNOS in particular) and the modem linked to the Internet via USB UMTS/HSDPA. To detect coverage, vehicle has pursued the routes of the Rome City Urban Bus Lines in each session as shown in Fig. 4, whereas few test results/samples have been taken away from several regions as mentioned below:

- Around Ponte Principe Amedeo Savoia Aosta, Roma.

- Via Nazionale and Piazza della Repubblica, Roma.

- Corso Vittorio Emanuele II, 00186 Roma, Italy.

After configuring the GPS Receiver Origin ORG1300 to facilitate the SBAS messages, activates the corresponding corrections settlement and yields at least the message GGA (NMEA) positioning with a period equal to 1 second. Along the desired route, the PC has sampled the data generated by the GPS receiver (the manifestation of the GPS /EGNOS positioning and messages) and executed data from adjoining locations and record/trace it with a remote control center via Internet. The successful transaction of data seems to be achievable when network signals exist along the route. The corresponding Fig. 5 indicates the precise real time track during the experiment.

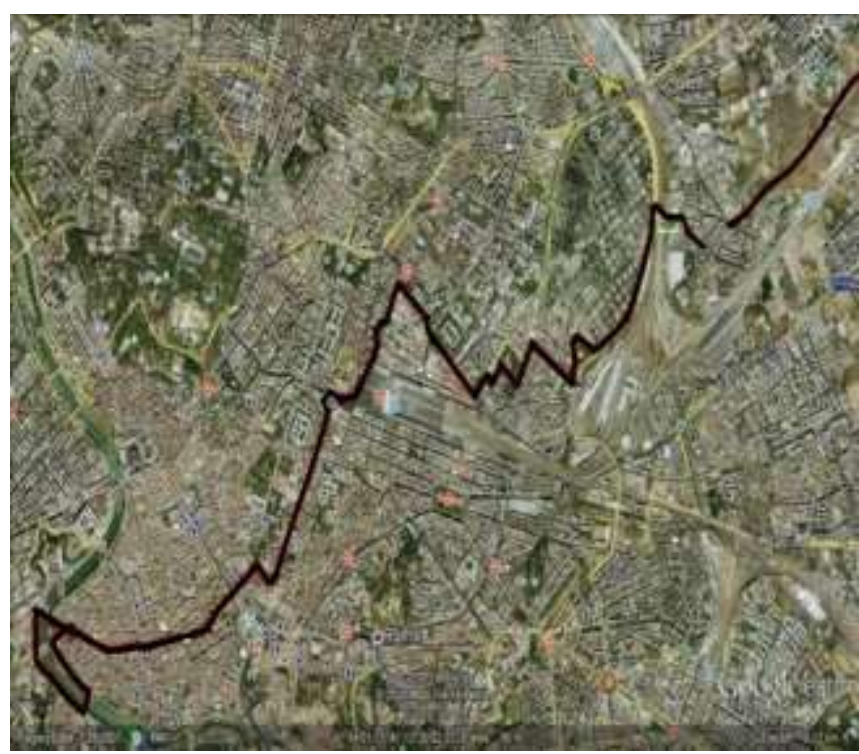

Fig. 5. Accredited Track Pursued Thru the Experiment.

\section{Test Analysis of Position Calibration}

The Wireless Internet Connection has been utilized to acquire EDAS messages and boosting the accuracy of the localization in urban areas.

A. Performance Comparison between Solitary GPS System and Mutual GPS + EGNOS Signals when Latitude is Not Highly Mounted.

The resultant sample around the area of Ponte Principe Amedeo Savoia Aosta Roma indicates the comparison of solitary GPS and collaborative GPS+EGNOS performance in Fig. 6 and Fig. 7.

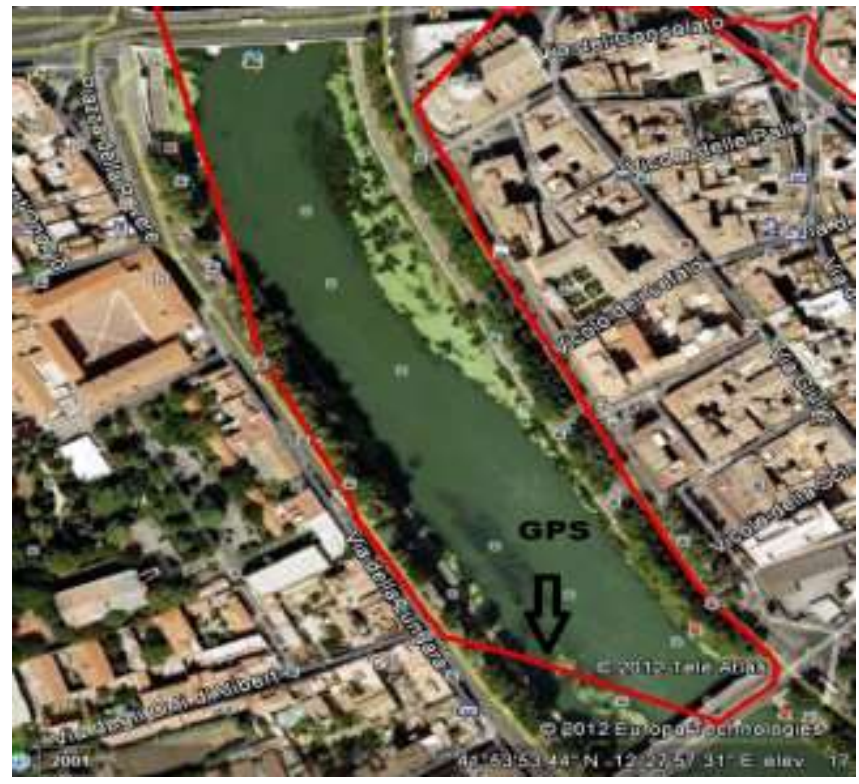

Fig. 6. Solitary GPS Signal Performance. 


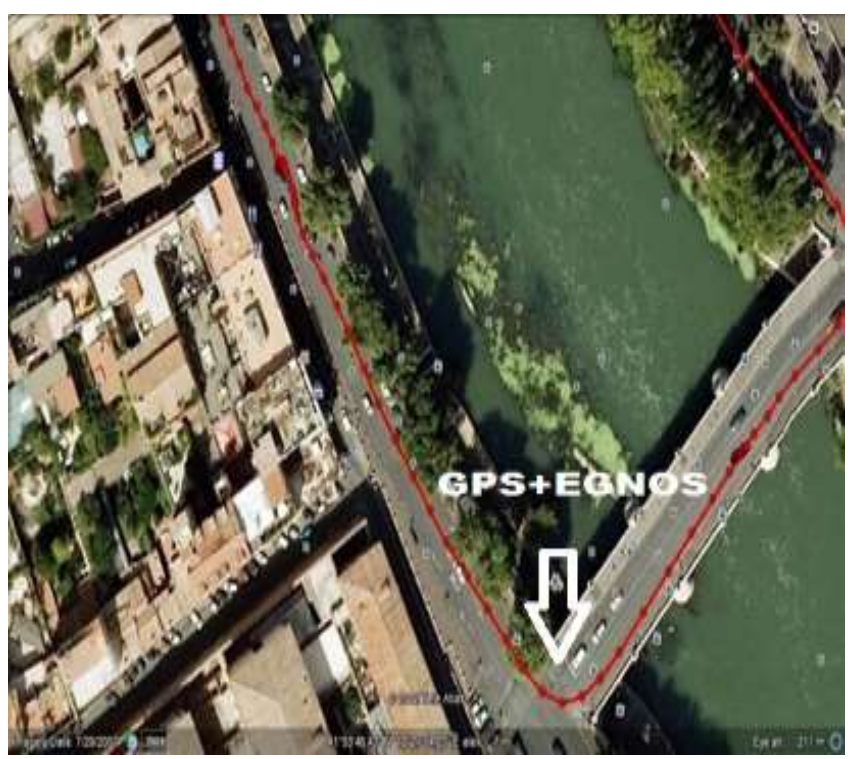

Fig. 7. GPS + EGNOS Signal.

\section{B. Evaluate the Percentage of Time in which EDAS could Achieve}

The experimentation has been analysed which estimates the percentage of available network service and their respective delays such as joint GPS+EGNOS signals and solitary GPS as shown in Fig. 7.

- Blue bar represents the $91.89 \%$ availability intervals of EDAS services.

- EDAS and GPS comparative accessibility generates 91.10\% (Orange Bar Stats).

- Availability of EGNOS service $42.26 \%$ suffered due to inaccuracy in Urban Territory (Specified by Gray Bar).

Though, the availability of EGNOS is quite modest with $42.26 \%$ due to urban region obstacles and mega structures as shown in Fig. 5 where accuracy of EGNOS starts to drop and metropolitan canyon disturb precision. However, it harvests astonishing outcomes with improved accuracy when open terrain regions are tested as indicated in Fig. 7.

In the category of satellite navigation augmentation systems, EGNOS enhances the precision of GPS by delivering a positional correctness within three meters. Moreover, GPS receiver without EGNOS measure positional data within 17 meters. Furthermore, the navigation system offers statistics about precision of the location, which is related to the trust developed due to validation of the system's integrity by EGNOS. In addition, it also delivers information about suitable usage of navigation system by activating timely warnings. Integrity feature ensures the accurate tracking of the location in case of emergency situations such as aeronautics and maritime circumstances.

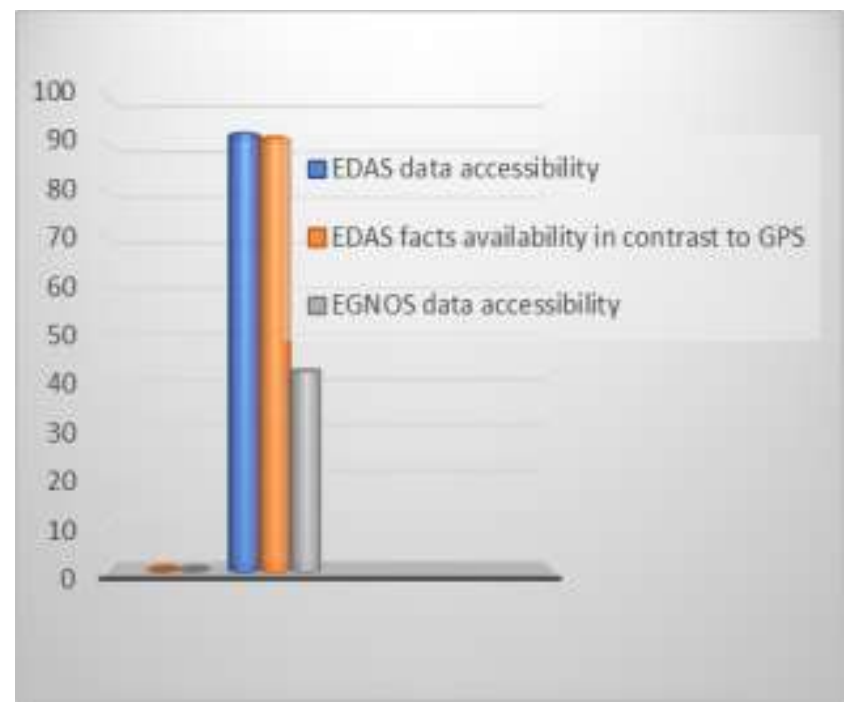

Fig. 8. Availability of Service Representing Scrutinized Networks (In Percentage).

Conversely, EDAS is far more reliable in Urban areas with availability of $91.89 \%$ as shown in Fig. 8. Furthermore, EDAS can assist in those spots where the EGNOS and GPS signals are not visible. During tests, it is investigated that attainment of both GPS+EGNOS corrections can detect accurate positions are detected but still GPS+EGNOS signals lags at some locations such as under the tunnels/bridges where there are no visible satellites.

In upcoming future, there are two possibilities for accessibility of best positions - uplift the accuracy of position either by SISNeT or EDAS, especially for those places where the signals are weak or not available. However, SISNeT does not provide safety of life while EDAS is designed in such a way as to provide accuracy as much as the safety of life.

\section{CONCLUSION}

In this paper, the comparative analysis of EGNOS and GPS has been accomplished by accumulating the data on diverse locations of Rome City. It has been witnessed that EGNOS is extremely accurate in timberlands with less congestion in outcomes. However, EGNOS downtown analysis submits inaccuracy in measurements due to hindrances such as tall buildings, bridges, trees and metallic obstructions in the vehicle. With GPS tests, it has been scrutinized that GPS constellation in medium orbits remains unobstructed globally and suits more to urban areas as influence of obstacles seems to be . In open plateaus, though, EGNOS is more precise in contrast to GPS with available data positions. Currently, Pakistan is substantially lacking its own Augmentation System and relying on the American GPS Systems (global accuracy of 10-15 meters) to stipulate statistics about Route Guidance in Pakistan Territory. Though, space agency of Pakistan- SUPARCO have joined their hands with P.R. China to launch their own augmentation system in the upcoming days. 


\section{ACKNOWLEDGMENT}

This research work has been carried out in the Rome City Center by ensuing number of bus routes and steered on-board a car. We would like to thank Prof. Dr. Ernestina Cianca for her indispensable guidance and assistance throughout the project as without her ideas stream of research tasks were not attainable. The author would also like to thank Dr. Riaz Ahmed Soomro for the guidance.

\section{REFERENCES}

[1] European Global Navigation Satellite Systems Agency (GSA), "EGNOS Service Definition Document, Safety of Life Service (SoL) Issue 3.0, ISBN: 978-92-9206-025-1 can be accessed via: https://egnosportal.gsa. europa.eu/sites/default/ files/uploads/Brochure_SoL2015_150924_High Def.pdf.

[2] Kaplan, Elliott D. and Hegarty, Christopher, "Understanding GPS: Principles and Applications", Second Edition, Artech House Publishers, 2005.

[3] Felski, Andrzej, Aleksander Nowak, and Tomasz Woźniak. "Accuracy and availability of EGNOS-results of observations." Artificial Satellites 46, no. 3 (2011): 111-118.

[4] Angrisano, A., S. Gaglione, and C. Gioia. "Performance assessment of GPS/GLONASS single point positioning in an urban environment."Acta Geodaetica et Geophysica 48, no. 2 (2013): 149-161.

[5] Zumberge, J. F., M. B. Heflin, D. C. Jefferson, M. M. Watkins, and F. $\mathrm{H}$. Webb. "Precise point positioning for the efficient and robust analysis of GPS data from large networks." Journal of geophysical research: solid earth 102, no. B3 (1997): 5005-5017.

[6] Héroux, P., Y. Gao, J. Kouba, F. Lahaye, Y. Mireault, P. Collins, K. Macleod, P. Tétreault, and K. Chen. "Products and applications for
Precise Point Positioning-Moving towards real-time." In Proceedings of the 17th international technical meeting of the satellite division of The Institute of Navigation (ION GNSS 2004), pp. 1832-1843. 2004.

[7] El-Mowafy, Ahmed, Manoj Deo, and Nobuaki Kubo. "Maintaining realtime precise point positioning during outages of orbit and clock corrections." GPS solutions 21, no. 3 (2017): 937-947.

[8] El-Mowafy, Ahmed, and Nobuaki Kubo. "Integrity monitoring for Positioning of intelligent transport systems using integrated RTK-GNSS, IMU and vehicle odometer." IET Intelligent Transport Systems 12, no. 8 (2018): 901-908.

[9] Specht, Cezary, Jan Pawelski, Leszek Smolarek, Mariusz Specht, and Pawel Dabrowski. "Assessment of the positioning accuracy of DGPS and EGNOS systems in the Bay of Gdansk using maritime dynamic measurements." The Journal of Navigation 72, no. 3 (2019): 575-587.

[10] Grunwald, Grzegorz, Mieczysław Bakuła, Adam Ciećko, and Rafał Kaźmierczak. "Examination of GPS/EGNOS integrity in north-eastern Poland." IET Radar, Sonar \& Navigation 10, no. 1 (2016): 114-121.

[11] Augmentation and integrity monitoring network and EGNOS Performance Comparison for Train Positioning. IEEE Signal Processing Conference (EUSIPCO), 2014 Proceedings of the 22nd European. Date of Conference: 1-5 Sept. 2014. Electronic ISSN: 2076-1465.

[12] Simo Marila; Mohammad Zahidul H. Bhuiyan; Jaakko Kuokkanen; Hannu Koivula; Heidi Kuusniemi.- Performance Comparison of Differential GNSS, EGNOS and SDCM in Different User Scenarios in Finland IEEE 2016 European Navigation Conference (ENC).

[13] EGNOS Open Service (OS) Service Definition Document, EGNOS Open Service (OS) SDD, Issue 2.1. Accessed via: https://egnosportal.gsa.europa.eu/sites/default/files/EGNOS_OS_SDD_2.1.Pdf

[14] Egnos Data Access Service (EDAS) Service Definition Document, SDD, Issue 2.0, April 2013, EGN-SDD EDAS, V2.0. Accessed via: http://www.galileoservices.org/news_events/edas_sdd_v2_0.pdf. 